\title{
Cross-regional emergency scheduling planning for petroleum based on the supernetwork model
}

\author{
Tao $\mathrm{Lv}^{1,2} \cdot$ Yan $\mathrm{Nie}^{2,3} \cdot$ Chun-Ling Wang ${ }^{4} \cdot$ Jian $\mathrm{Gao}^{2}$
}

Received: 9 October 2017 / Published online: 23 June 2018

(C) The Author(s) 2018

\begin{abstract}
Petroleum, the most important energy source in the world, plays an essential role in securing economic development. If a petroleum shortage happens, it will severely disrupt production and life. Cross-regional emergency scheduling can effectively alleviate a petroleum shortage and further enhance the efficiency of the emergency response. Considering the general lack of focus on cross-regional petroleum dispatching management, we propose a three-layer emergency scheduling network for petroleum based on a supernetwork model that can increase the regional emergency correlation by adding a transfer management process. Then, we compare the total demand for petroleum and the emergency costs considered in the petroleum emergency scheduling supernetwork model (the single-region and the cross-region scenarios). The result shows that the cross-regional emergency scheduling pattern can effectively enhance the efficiency of the emergency preparations and reduce the emergency costs in most cases. However, when the vulnerabilities in the crossregional link grow or the regional linkage decreases, the effect of single-regional scheduling is better. In addition, the advantages of the cross-regional emergency scheduling network will be strengthened with an increase in its maximum emergency capability. Nonetheless, this advantage will disappear when the petroleum demand in the crisis layer reaches the maximum emergency response capacity. Finally, according to the comparative analysis simulation among scenarios, certain strategic policy recommendations are suggested to improve the petroleum emergency scheduling ability in regions. These recommendations include strengthening the cross-regional coordination mechanism, increasing the modes of petroleum transportation and enhancing the carrying capacity of regional emergency routes.
\end{abstract}

Keywords Petroleum emergency $\cdot$ Supernetwork $\cdot$ Cross-regional scheduling $\cdot$ Scheduling strategy $\cdot$ Variational inequality

\section{Introduction}

Edited by Xiu-Qin Zhu

Handling editor: Qi Zhang

Yan Nie

nieyan1988@163.com

1 Jiangsu Energy Economy and Management Research Base, China University of Mining and Technology, Xuzhou 221116, Jiangsu, China

2 School of Management, China University of Mining and Technology, Xuzhou 221116, Jiangsu, China

3 School of Civil Engineering, LanZhou University of Technology, Lanzhou 730050, Gansu, China

4 Department of Commerce and Trade, Xuzhou Vocational Technology Academy of Finance and Economics, Xuzhou 221008, Jiangsu, China
Global petroleum demand is continually increasing and petroleum continues to represent a high proportion of energy consumption and is regarded as one of the most important resources in the world (British Petroleum 2015). Petroleum shortages, such as those caused by serious natural disasters, war or security incidents, will bring on an energy crisis that will negatively impact on normal social processes and life. The petroleum shortage emergency scheduling system can effectively ameliorate the problem of a petroleum shortage in a certain region. The USA, Germany and other developed countries have already built petroleum shortage emergency management systems and have enacted relevant management laws. However, China, the world's second largest petroleum consumer after the USA (Asia Pacific Energy Research Center 2015), has not established an effective petroleum shortage emergency 
mechanism or a shortage emergency management department. In addition, the seriously unequal distribution of petroleum and the large gap in the consumption capacities between different regions highlight the regional conflicts of petroleum supply and demand in China (Lu and Gao 2016). Therefore, it is necessary to propose a petroleum shortage emergency scheduling system to alleviate the regional imbalance of the petroleum supply, especially in China.

China has experienced several petroleum shortage incidents. For example, in 2008 in China, Sichuan experienced a petroleum shortage when the railway was interrupted by the Wenchuan earthquake. Then, the government implemented emergency petroleum transportation from Chongqing, Gannan and the northeastern reserves to ensure an adequate supply for disaster relief (Jiang 2008). In October 2010, China eliminated the "diesel shortage". The China National Petroleum Corporation (CNPC) transported petroleum from Dalian to relieve the market pressure (NetEase Financial News 2001). These above cases show two features of petroleum shortage emergencies. On one hand, petroleum shortage incidents are characterized by wide spread, shorter emergency response times. On the other hand, cross-regional emergency scheduling could effectively alleviate and improve the efficient handling of petroleum shortage emergencies.

Although some research on petroleum emergency management has emerged, most studies mainly focus on the supply chain optimization, resource allocation and emergency supply analysis, which concentrated on the technical innovations of petroleum emergencies and the optimization of petroleum supply, shortage and distribution. There is less focus on cross-regional emergency scheduling. To fill this gap, this work proposes an emergency scheduling model for cross-regional petroleum management. It describes the internal coordination mechanism in the petroleum emergency deployment process and increases the regional association behaviour. First, we propose a three-layer supernetwork model of petroleum emergency scheduling using the quantitative expression of objective functions and decision-making behaviour that overemphasizes the importance of cross-regional emergency scheduling. Using the variance inequality and the modified projection algorithm, we then analyse the dispatching characteristics of the emergency scheduling supernetwork model in both a single-region and cross-region scenario. The results show that the cross-regional cooperative response could improve the efficiency of petroleum emergency response and reduce its costs. Finally, according to the research results, we find that increasing the modes of petroleum transportation and enhancing the carrying capacity of regional emergency routes could effectively alleviate petroleum shortage emergencies and ensure the security of the petroleum supply.

\section{Literature review}

A disruption of the petroleum supply will seriously affect national economic activities and trigger panic. Therefore, the emergency management of any petroleum supply shortage is a broad concern. Iakovou (2001) first proposed the petroleum emergency management theory to address the maritime transportation of petroleum products. In addition, the supernetwork is commonly used to address the complicated systematic problems characterized by numerous nodes and network nesting. Nagurney et al. (2002), a scientist in the USA, stated that a supernetwork was better than any existing network. Accordingly, the supernetwork is widely applied in transportation control, supply chain management and internet financial analysis. Considering the features of logistics transportation and economic circulation in petroleum cross-regional emergency scheduling, we construct a three-layer cross-regional emergency scheduling model based on the supernetwork theory. Therefore, we introduce research results from two aspects: the petroleum emergency management and the application of a supernetwork.

\subsection{Petroleum emergency management}

Recent studies on the petroleum shortage emergency management highlight the problems for path optimization in emergency transmission and distribution (MirHassani 2008), coordinated resource allocation (Fernandes et al. 2013), emergency supply analysis (Chen et al. 2013; Bai et al. 2016), and technical innovations of petroleum emergency management (Abdalla and Alharbi 2017; Sha et al. 2017). Specifically, Jiang (2008) analysed the emergency strategies after the Wenchuan earthquake in China and found that the petroleum emergency agents lacked coordinating mechanisms. Hence, based on the lack of coordination, Jiang used the Floyd algorithm to optimize the "shortest path" problem for determining the optimal emergency scheduling programme that could improve the coordination (Jiang 2013). He later constructed a model of a refined oil emergency transmission and distribution system that could further enhance the coordination ability of a refined oil emergency agent (Jiang et al. 2014). In addition, for the Indian Jaipur storage terminal accident, Sharma et al. (2015) proposed an emergency response plan for petroleum product storage terminals to improve coordination in crisis management.

In addition to coordination mechanism research, safety evaluation and path optimization have always been the focus of petroleum shortage emergency scheduling. To effectively limit and control oil spills, Lyu et al. (2014) established a risk assessment model for offshore petroleum 
activities and effectively prepared petroleum spill response resources. Ambituuni et al. (2015) constructed a safety risk assessment framework for the road transportation of petroleum in developing countries and found that improving government regulations could effectively reduce the risk. Kazemi and Szmerekovsky (2015) proposed a mixed integer linear programming model to reduce the petroleum production and distribution costs by optimizing the petroleum supply chain. Thereafter, Oliveira et al. (2016) established a stochastic optimization model of origin-toterminal refineries to ensure the fundamental supply of crude oil. Based on two realistic military contingency events in Mali and Japan, Othman et al. (2017) presented a multi-agent management system of petroleum emergency supply to realize the effective coordination of emergency transportation. It was widely believed that the technical innovations of petroleum emergency management could alleviate petroleum shortages. Abdalla and Alharbi (2017) applied geospatial information systems (GIS) to petroleum emergency management, and Sha et al. (2017) applied cloud technology to petroleum emergency management to find an efficient emergency management mode for solving the temporary emergency management model. Considering the multiple sources of LNG, Zhang et al. (2017) proposed a three-stage stochastic programming model for LNG supply system infrastructure development and inventory routing in countries.

However, these studies mainly solve the emergency logistics transportation problem for a single region (such as providing optimal supply and reducing emergency costs) and promote the emergency technology. The characteristics of cross-regional, multi-attribute, network nesting and emergency modes have garnered minimal focus. Therefore, we propose a supernetwork model with multi-level and multi-attribute and dynamic characteristics of petroleum shortage emergency scheduling that could optimize emergency response to reduce the costs.

\subsection{Application of supernetwork}

A supernetwork is a combination of multiple nested networks and has been widely applied to transportation, logistics and other fields. For example, Liu and Nagurney (2013) implemented global outsourcing and rapid production strategies to achieve a supernetwork supply chain balance. Yamada et al. (2011) found that the efficiency of supply chain networks would be significantly improved by transforming the "supply chain-transport" network into a supernetwork. In addition, a multi-state supernetwork was proposed to investigate the effects of land-use transportation scenarios on peoples' travel patterns (Liao et al. 2015). Therefore, a supernetwork is a promising tool to analyse the hierarchical structures of networks (Wang et al. 2016).
The supernetwork theory can also analyse the energy and information flow in the emergency management process in multiple dimensions, which can coordinate resource interactions. Zhu and Cao (2012) studied the interaction between the disaster risk environment and the dynamic allocation of emergency resources. They then presented the effects of risk on emergency resource demands and costs (Zhu and Cao 2015). Considering the complex characteristics in the whole process of emergency scheduling ("predisaster, disaster preparedness, disaster"), Zhu and Du (2015) proposed a supernetwork with a "natural association network", a "resources allocation network" and a "social relations network". The above research on the application of the supernetwork provides a theoretical basis for our proposed supernetwork model of petroleum crossregional emergency scheduling.

Based on the existing studies, we analyse the interaction pattern of resources and information in these petroleum shortage emergency events. Then, we propose a cross-regional petroleum emergency scheduling model based on the supernetwork theory that can enhance the efficiency of the emergency measures and reduce the emergency costs in most cases. First, the cross-regional scheduling framework of a petroleum emergency is constructed by clarifying the influencing factors of emergency dispatch. Then, we analyse the changes of emergency volume and emergency costs in the petroleum emergency supernetwork model for both a single region and the cross-regional scenario. Finally, according to the influence on the cross-regional emergency scheduling, we propose some policy recommendations to effectively enhance the entire petroleum emergency management system.

\section{Analysis of the supernetwork characteristics and structure}

\subsection{Supernetwork characteristics of the cross- regional emergency scheduling for petroleum}

A supernetwork is characterized by multiple agents, multiple attributes, multiple criteria, congestion and a dynamic nature (Wang and Wang 2008). Similarly, the cross-regional scheduling system for a petroleum shortage emergency also maintains these typical supernetwork structure features.

(1) Multiple agents. On one hand, the agents of a crossregional emergency scheduling system for petroleum shortage include the government, the market and the social organization, which work together to build a steady emergency management system. On the other 
hand, the agents' function is different in each stage of emergency management. Specifically, the different emergency agents cooperate to formulate the petroleum scheduling programme in the early stage; when entering the medium term, they interactively complete the coordinated allocation of petroleum. The agents ultimately improve the regional emergency pre-plan for petroleum.

(2) Multiple levels. The cross-regional scheduling system for a petroleum shortage emergency consists of three levels: rescues, transfer and crisis. In addition, the rescues and transfer levels will present multiple level features.

(3) Multiple attributes. The emergency capacity of each region is related to the population density, the economic level, the path pattern, the emergency awareness, and the integrity of the emergency system. Due to the difference of these factors in various regions, the contingency plans and the emergency drills for petroleum are also uneven.

(4) Multiple criteria. The petroleum shortage emergency scheduling process needs to consider certain criteria, such as emergency time, emergency cost, satisfaction, security and environmental impact. Among these criteria, the emergency time measures the timeliness of the resources' arrival; the emergency cost mainly refers to the petroleum transportation expense. The satisfaction reflects the extent of satisfaction for each crisis region; the security includes the robustness of the scheduling network and the stability of the road traffic. The environmental factors are concerned with the emergency path and other harsh environments.

(5) Congestion. The petroleum supply disruption event is characterized by rapid and wide dispersion; however, the congestion phenomenon aggravates the loss and asymmetry of the emergency information in the cross-regional emergency scheduling network.

(6) Dynamics. The emergency scheduling activity will show dynamic change characteristics during the whole petroleum emergency process. Correspondingly, the decision-making target in each stage will change with the different emergency tasks.

Therefore, a supernetwork provides a suitable tool for our study of cross-regional scheduling for a petroleum shortage emergency, which breaks through the limitation of the homogeneity hypothesis on the single "point" and the single "edge" in an emergency network. Furthermore, a supernetwork can describe the coordination mechanism of different networks with distinguished attributes using quantitative analysis.

\subsection{Supernetwork structure of the emergency scheduling for petroleum}

In this paper, the process of cross-regional emergency scheduling for petroleum is highly abstracted into a network with three levels: rescues, transfer and crisis. We construct a coordinated supernetwork structure for the petroleum emergency by exemplifying regions $\mathrm{A}$ and $\mathrm{B}$. The significant emergency agents are described as nodes in this emergency scheduling network, including the rescue points $(\mathrm{R})$, the transfer points $(\mathrm{T})$ and the crisis points $(\mathrm{C})$. In addition, the directed edge shows the scheduling quantity of petroleum in the single region and the cross-regions, which are marked with solid lines. In contrast, the coordination relationship between two regions is represented by a dashed line. These lines are shown in Fig. 1.

In the above supernetwork structure, there is vertical cooperation from the rescue points to the crisis points between the different levels, while there are certain horizontal competitions in the same level (the path selection of transfer points). Furthermore, a command centre $O$ for the coordination of cross-regional emergency scheduling is established, which can ensure cross-regional relief for each crisis point.

In view of the complexity of emergency cooperation in the different regions, we make the following assumptions to facilitate the construction of the supernetwork model: (1) The petroleum for supply and relief in each region is homogeneous. (2) The emergency command centre provides instructions for a rescue point to allocate the petroleum resources to the crisis point. (3) The total petroleum in the rescue point is greater than the total demand for petroleum in the crisis point. (4) All the emergency agents are rational; they are on the basis of satisfying the petroleum shortage, utilizing the reduction in the emergency cost and the emergency time as the main purpose. (5) The functions of emergency costs and emergency time are all continuously differentiable convex functions (Wang and Wang 2008).

\section{Model}

The supernetwork modelling of a cross-regional emergency scheduling system is conducted from two aspects: the optimization of petroleum emergency scheduling and the optimal behaviour of decision-making in the rescue points. In addition, the constructed supernetwork model is flexible and dynamic, which is embodied by adding a transfer layer to coordinate the cross-regional association in emergency process. 


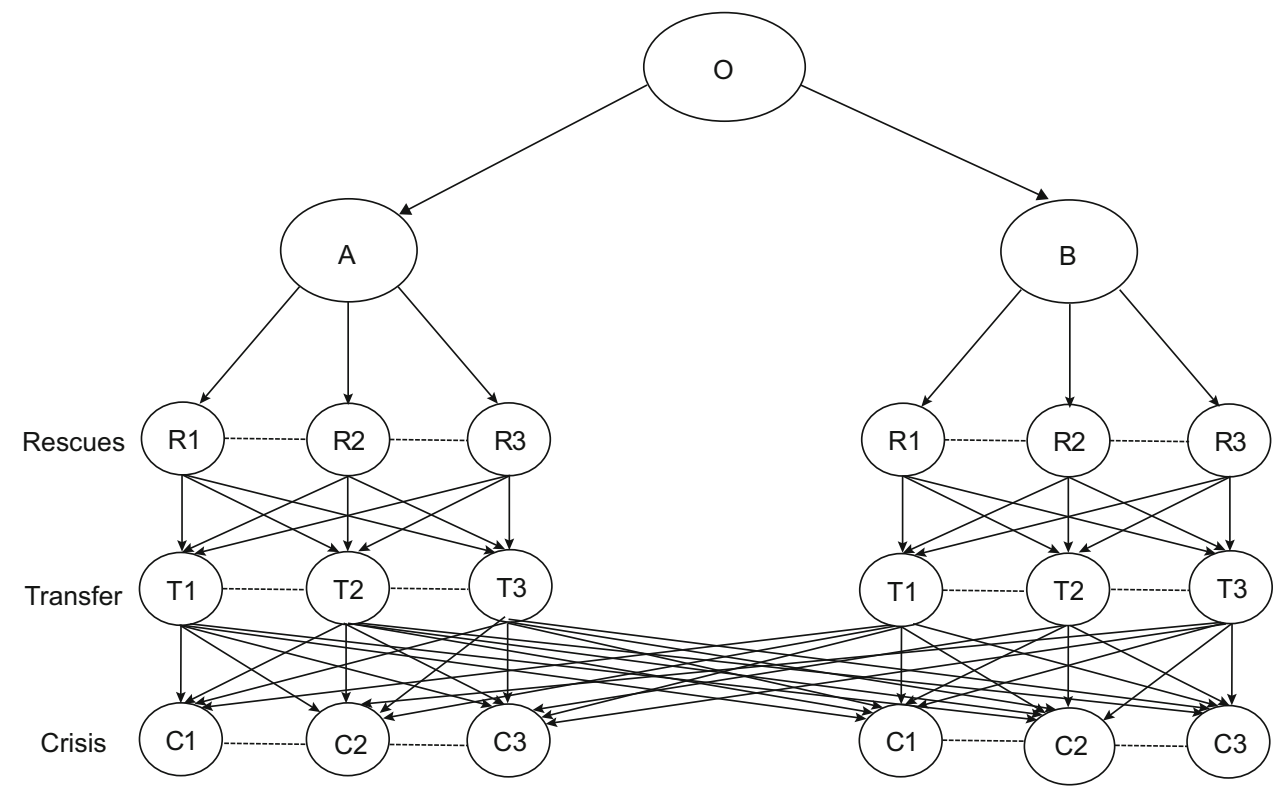

Fig. 1 Cross-regional emergency scheduling supernetwork structure of petroleum

\subsection{Construction of the supernetwork model}

The optimization objective of a petroleum cross-regional emergency scheduling supernetwork is to determine the appropriate allocation from the rescue points to the crisis point through the transfer point. Moreover, this model will ensure the lowest emergency costs and time. The new generalized optimization function for the cross-regional emergency scheduling of petroleum is constructed by transforming the multi-objective function into a single function according to the linear weight method, as shown in Formula (1).

$$
\begin{gathered}
\min \sum_{l \in L} g_{l}\left(f_{l}, v_{l}, r_{l}\right)=\alpha_{1} \sum_{l \in L} c_{l}\left(f_{l}, v_{l}, r_{l}\right)+\alpha_{2} \sum_{l \in L} t_{l}\left(f_{l}, v_{l}, r_{l}\right) \\
\text { s.t. } \quad f_{l} \leq s_{l}, \forall l \in L \\
f_{l}=\sum_{p \in P} x_{p} \delta_{l p}, \forall x_{p} \geq 0 \\
G_{p}=\sum_{l \in L} g_{l} \delta_{l p}
\end{gathered}
$$

where $l$ is a random connection line in the network, $L$ is the set of all the lines, and $l \in L . p$ is an arbitrary path connecting the start point and the termination point. In addition, $P$ is the set of the total path with $p \in P . v_{l}$ and $r_{l}$, respectively, represent the vulnerability and the correlation degree of each line. $f_{l}$ is the volume of emergency resources in the line $l, s_{l}$ is the maximum emergency capability of the line $l$, and $x_{p}$ is the volume on the path $p$. In addition, $\delta_{l p}$ is used to determine whether or not the line $l$ is included in the path $p$. If $l$ is included in the path $p$, $\delta_{\mathrm{l} p}=1$; otherwise, $\delta_{l p}=0$.

The emergency scheduling cost function is $c_{l}\left(f_{l}, v_{l}, r_{l}\right)$, which includes the emergency volume, the emergency path vulnerability and their correlation of the line $l$. Similarly, the scheduling time function is $t_{l}\left(f_{l}, v_{l}, r_{l}\right)$ and is expressed with the same variables. $g_{l}\left(f_{l}, v_{l}, r_{l}\right)$ is a generalized emergency cost function on the line $l . \alpha_{1}$ and $\alpha_{2}$, respectively, represent the weights of the transportation and time costs in the emergency scheduling, while $\alpha_{1}+\alpha_{2}=1$. However, in the generalized emergency function associated with the path $p, G_{p}=\sum_{l \in L} g_{l} \delta_{l p}$ is summed by the generalized emergency costs of all the lines that comprise the path.

\subsection{Optimal behaviour of the emergency response based on the Wardrop principle}

It is difficult to determine the best rescue and crisis point with a quantifiable optimal goal. Therefore, the petroleum emergency command centre tends to maintain balance between the scheduling quantity and the emergency costs from the rescue points to the crisis point. This paper analyses the emergency scheduling of petroleum due to the users' optimal behaviour in the traffic network equilibrium [Wardrop principle (Nagurney 2006; Zhu and Du 2015)].

$G_{p}\left(x_{p}^{*}\right)-\lambda_{w}^{*}\left\{\begin{array}{l}=0, x_{p}^{*}>0 \\ \geq 0, x_{p}^{*}=0\end{array}\right.$

where $w$ means a couple of starts and terminations in the network, and $w \in W$. However, $\lambda_{w}$ is the failure cost in a certain $w$, that is, the minimum relief costs. In addition, 
Eq. (3) indicates that the criterion for selecting the optimal emergency route is to obtain the minimum emergency costs. The emergency path will be activated only if the generalized emergency cost $G_{p}\left(x_{p}\right)$ is equal to the minimum emergency costs on the path $p$. In contrast, the allocation of petroleum on the path $p$ can be described as $x_{p}=0$.

The following relationship exists between the demand for resources at the crisis level, the minimum emergency costs and the total amount of petroleum that is urgently scheduled through each path (Zhu and Du 2015).

$\sum_{p \in P_{w}} x_{p}^{*} \begin{cases}=d_{w}\left(v_{w}, \lambda_{w}^{*}\right), & \lambda_{w}^{*}>0 \\ \geq d_{w}\left(v_{w}, \lambda_{w}^{*}\right), & \lambda_{w}^{*}=0\end{cases}$

$P_{w}$ is a total set of $w . p \in P_{w} . d_{w}\left(v_{w}, \lambda_{w}\right)$ is the demand for petroleum in $w$, assuming that $w$ is related to the emergency capability $v_{w}$ and the minimum costs of the rescue points $\lambda_{w}$. $v_{w}$ reflects the comprehensive emergency response capacity of the rescue points, which is generally affected by the population density in the crisis point, the emergency awareness, the probability of petroleum shortages, the ensuing consequences and other factors (Zhu and Du 2015).

The necessary conditions for which the crisis point can obtain relief are shown in Formula (4). If the rescue point decides to allocate the petroleum, the total supply must meet the total demand. The emergency action will terminate if supplies are less than demand, and the minimum relief costs are $\lambda_{w}=0$.

\subsection{Model solving}

According to hypothesis (4), the constructed model is a convex optimization problem that is continuous and differentiable. It can be transformed into the corresponding variational inequality to find the solutions. Using the Lagrangian multiplier $\beta_{l}$ and the volume constraint $f_{1} \leq s_{l}$, the final generalized optimization objective function can be transformed into the following variational inequalities:

$$
\begin{aligned}
& \sum_{p \in P}\left(\alpha_{1} \frac{\partial C_{p}}{\partial x_{p}}+\alpha_{2} \frac{\partial T_{p}}{\partial x_{p}}+\sum_{l \in L} \beta_{l} \delta_{l p}+G_{p}-\lambda_{w}\right) \times\left(x_{p}-x_{p}^{*}\right) \\
& +\sum_{l \in L}\left(s_{l}-\sum_{p \in P} x_{p} \delta_{l p}\right) \times\left(\beta_{l}-\beta_{l}^{*}\right)+\left(\sum_{p \in P_{w}} x_{p}-d_{w}\left(v_{w}, \lambda_{w}\right)\right. \\
& \times\left(\lambda_{w}-\lambda_{w}^{*}\right) \geq 0 \quad\left(\forall\left(x_{p}, \beta_{l}, \lambda_{w}\right) \in K\right)
\end{aligned}
$$

where $K \equiv\left\{\left(x_{p}, \beta_{l}, \lambda_{w}\right) \mid x_{p} \geq 0, \beta_{l} \geq 0, \lambda_{w} \geq 0, \forall p, l, w\right\}$, $C_{p}=\sum_{l \in L} c_{l} \delta_{l p}$ is the scheduling costs, and $T_{p}=$ $\sum_{\mathrm{l} \in L} t_{1} \delta_{\mathrm{l} p}$ is the time costs on the path $p$.

When the generalized emergency costs and the petroleum demand in shortage regions meet the requirements of the optimization function, the entire cross-regional scheduling network for petroleum emergency will achieve equilibrium. Furthermore, the volume of each emergency path in the network and the minimum relief costs in each crisis point should achieve equilibrium.

\section{Simulation result}

Numerical simulation analysis of the cross-regional emergency scheduling model is conducted by the following steps. First, we discuss the effects of key factors in the single-region condition. Then, compared with the singleregion scenario, we analyse the changes of emergency volume, costs and linkages in the cross-regional scenario. Finally, we analyse the influence of treatments with different attributes on emergency dispatch.

Assume that there are two regions, $\mathrm{A}$ and $\mathrm{B}$. Each region contains a rescue point, a transfer point, two crisis points, and five emergency paths. We can describe the structure of the supernetwork as shown in Fig. 2. The petroleum from regions $\mathrm{A}$ and $\mathrm{B}$ is transported to the crisis points through transfer points, which realize the cross-regional emergency scheduling. Furthermore, the number of rescue points, transfer points and crisis points can all be extended to a larger scale. However, in this work, the numbers of emergency agents are set lower for simpler calculations, which has no effect on the analysis of more complex crossregional emergency scheduling modes with more emergency agents and more complex emergency relationships.

Considering the importance of time and transportation costs in a petroleum emergency event, we set time costs slightly higher than transport costs and determined a functional expression by referring to the literature (Zhu and

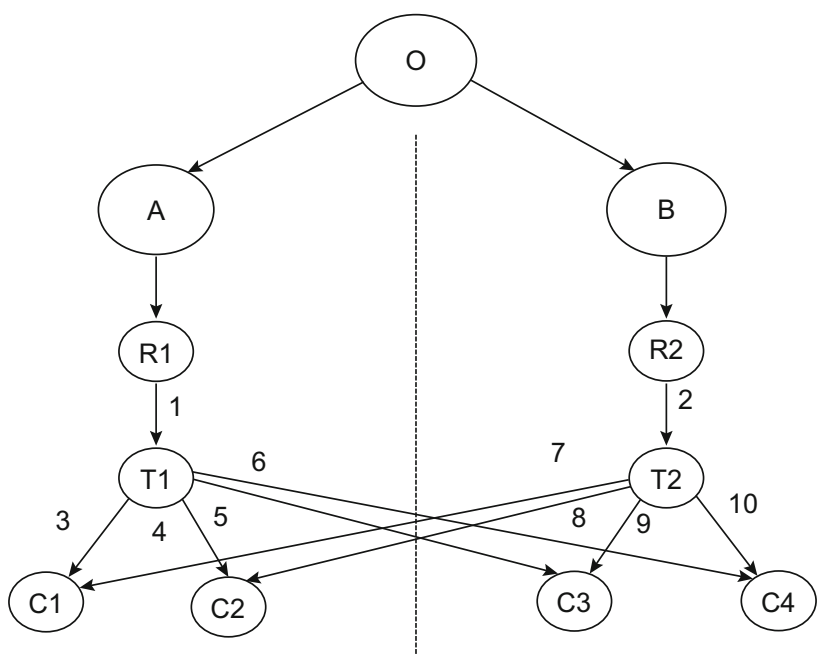

Fig. 2 A simulation example of a petroleum cross-regional emergency scheduling supernetwork 
Cao 2012). Moreover, the demand function of petroleum between the beginning and end points is set as $d_{w}\left(v_{w}, \mu_{w}\right)=v_{w}-\lambda_{w} \quad(\mathrm{Zhu}$ and $\mathrm{Du}$ 2015), where $v_{w 1}=200, v_{w 2}=400, v_{w 3}=200, v_{w 4}=400$, the weights of transportation costs and time costs are $\alpha_{1}=\alpha_{2}=0.5$, the Lipschitz constant is $\gamma=100$, the condition of variable convergence is $\left\|x^{k}-\max \left\{x^{k}-F\left(x^{k}\right), 0\right\}\right\| \leq 0.001$, and the initial values are all set 100 (as shown in Table 1). In addition, we only compare these dimensionless numerical values through simulation without considering their variable units. The objective function is input into the optimal condition of the supernetwork equilibrium and solved with MATLAB.

\subsection{Comparative analysis of the emergency scheduling in single region and cross- regional mode}

The emergency paths in region A include paths 1, 3, and 4. The paths for emergency scheduling to two crisis points are $p_{1}=\{1,3\}$ and $p_{2}=\{1,4\}$. The path sets whose terminations are the two crisis points are $p_{\mathrm{w} 1}=\left\{p_{1}\right\}$ and $p_{\mathrm{w} 2}=\left\{p_{2}\right\}$. If regions $\mathrm{A}$ and $\mathrm{B}$ cooperate with each other in the petroleum emergency, they will form a cross-regional emergency system containing two rescue points, two transfer points, four crisis points, and ten emergency paths. The path sets whose terminations are the four crisis points are $p_{\mathrm{w} 1}=\left\{p_{1}, p_{5}\right\}, \quad p_{\mathrm{w} 2}=\left\{p_{2}, p_{6}\right\}, \quad p_{\mathrm{w} 3}=\left\{p_{3}, p_{7}\right\}$ and $p_{\mathrm{w} 4}=\left\{p_{4}, p_{8}\right\}$. Therefore, the sensitivities of the relevant parameters are as follows.

\subsubsection{Effects of different weights $\alpha$ on the supernetwork}

We investigate the impacts of weight on the emergency costs and time by setting the original weight $\alpha_{1}=\alpha_{2}=0.5$, which is then compared with the two extreme conditions $\alpha_{1}=0.2, \alpha_{2}=0.8$ and $\alpha_{1}=0.8, \alpha_{2}=0.2$ (Table 2).
In the single region emergency scenario, when $\alpha_{1}=\alpha_{2}=0.5$, the total scheduling quantity is 10.1 , the minimum relief costs are 1189.9, and the generalized emergency costs are 39.48. When the weights change to $\alpha_{1}=0.2, \alpha_{2}=0.8$, the indicators are 10.1, 1189.9 and 45.0 , respectively. When $\alpha_{1}=0.8, \alpha_{2}=0.2$, the indicators are 10.1, 1189.9 and 34.1, respectively. Compared with these results, we can observe that the weights of costs and time have minimal influence on the scheduling quantity and the minimum costs. However, it significantly affects the generalized emergency costs such as $T C_{2}<T C<T C_{1}$, showing that the emergency costs will increase if more emphasis is put on the response time in the cross-regional emergency management of petroleum. The change of the weight has no significant influence on the scheduling quantity and the minimum costs, but it obviously affects the generalized emergency costs. Specifically, it is more likely that the generalized emergency costs would increase if the petroleum emergency scheduling process is completed in a shorter time, since more funds will be spent on coordinating the transportation of the cross-regional petroleum in order to shorten the emergency time.

In the cross-regional emergency scenario, when $\alpha_{1}=\alpha_{2}=0.5$, the total scheduling quantity is 9.9 , the minimum relief costs are 1189.4, and the generalized emergency costs are 35.9, which are all less than those in the single region. Particularly, the reduction in generalized emergency costs is more than with the other two measures. Furthermore, we find that when $\alpha_{1}=0.8, \alpha_{2}=0.2$, its generalized emergency costs decrease by 5.2 , which is the largest decrease. However, its total scheduling quantity and minimum relief costs have the smallest decreases. No matter how the emergency costs and time weights change, the total scheduling of petroleum resources in the crossregional emergency dispatching mode are all less than that in the single-regional one. Furthermore, the minimum relief costs and the generalized emergency costs of the

Table 1 Parameter settings in simulation of cross-regional emergency scheduling supernetwork for petroleum

\begin{tabular}{|c|c|c|c|c|c|c|c|}
\hline Edge & Start point & Termination point & $c_{l}\left(f_{l}, v_{l}, r_{l}\right)$ & $t_{l}\left(\mathrm{f}_{l}, \mathrm{v}_{l}, \mathrm{r}_{l}\right)$ & $v_{l}$ & $s_{l}$ & $r_{l}$ \\
\hline 1 & Rescues 1 & Transfer 1 & $\left(v_{1}+0.4-0.1 r_{1}\right) f_{1}$ & $\left(2.6 v_{1}+1-0.5 r_{1}\right) f_{1}$ & $v_{1}=1$ & $s_{1}=5$ & $r_{1}=5$ \\
\hline 2 & Rescues 2 & Transfer 2 & $\left(2 v_{2}-0.1 r_{2}\right) f_{2}$ & $\left(2 v_{2}+1-0.1 r_{2}\right) f_{2}$ & $v_{2}=1$ & $s_{2}=5$ & $r_{2}=5$ \\
\hline 3 & Transfer 1 & Crisis 1 & $\left(v_{3}-0.2 r_{3}\right) f_{3}$ & $\left(v_{3}+0.2-0.1 r_{3}\right) f_{3}$ & $v_{3}=2$ & $s_{3}=4$ & $r_{3}=4$ \\
\hline 4 & Transfer 1 & Crisis 2 & $\left(v_{4}+1-0.5 r_{4}\right) f_{4}$ & $\left(2 v_{4}-r_{4}\right) f_{4}$ & $v_{4}=3$ & $s_{4}=2$ & $r_{4}=2$ \\
\hline 5 & Transfer 1 & Crisis 3 & $\left(v_{5}+1-0.1 r_{5}\right) f_{5}$ & $\left(2 v_{5}+2-0.2 r_{5}\right) f_{5}$ & $v_{5}=0$ & $s_{5}=5$ & $r_{5}=5$ \\
\hline 6 & Transfer 1 & Crisis 4 & $\left(v_{6}-r_{6}\right) f_{6}$ & $\left(v_{6}+1-r_{6}\right) f_{6}$ & $v_{6}=3$ & $s_{6}=1$ & $r_{6}=1$ \\
\hline 7 & Transfer 2 & Crisis 1 & $\left(2 v_{7}-r_{7}\right) f_{7}$ & $\left(2 v_{7}+1-r_{7}\right) f_{7}$ & $v_{7}=2$ & $s_{7}=3$ & $r_{7}=3$ \\
\hline 8 & Transfer 2 & Crisis 2 & $\left(2 v_{8}+1.5-0.5 r_{8}\right) f_{8}$ & $\left(v_{8}+2.5-r_{8}\right) f_{8}$ & $v_{8}=0$ & $s_{8}=2$ & $r_{8}=2$ \\
\hline 9 & Transfer 2 & Crisis 3 & $\left(v_{9}+1-r_{9}\right) f_{9}$ & $\left(2 v_{9}-r_{9}\right) f_{9}$ & $v_{9}=3$ & $s_{9}=4$ & $r_{9}=3$ \\
\hline 10 & Transfer 2 & Crisis 4 & $\left(v_{10}+1-0.5 r_{10}\right) f_{10}$ & $\left(2 v_{10}-r_{10}\right) f_{10}$ & $v_{10}=3$ & $s_{10}=2$ & $r_{10}=2$ \\
\hline
\end{tabular}


Table 2 Effect of the $\alpha$ in different scenarios

\begin{tabular}{|c|c|c|c|c|c|c|}
\hline \multirow[t]{2}{*}{$\alpha$} & \multicolumn{2}{|c|}{ Emergency scheduling quantity of petroleum } & \multicolumn{2}{|c|}{ The minimum relief cost } & \multicolumn{2}{|c|}{ Generalized emergency cost } \\
\hline & Single region & Cross-region & Single region & Cross-region & Single region & Cross-region \\
\hline$\alpha_{1}=\alpha_{2}=0.5$ & 10.1 & 9.9 & 1189.9 & 1189.4 & 39.5 & 35.9 \\
\hline$\alpha_{1}=0.2, \alpha_{2}=0.8$ & 10.1 & 9.9 & 1189.9 & 1189.9 & 45.0 & 39.8 \\
\hline$\alpha_{1}=0.8, \alpha_{2}=0.2$ & 10.1 & 9.9 & 1189.9 & 1189.9 & 34.1 & 32.0 \\
\hline
\end{tabular}

cross-regional emergency dispatching are also significantly less than the single-regional mode. Therefore, the crossregional emergency coordination mode shows a better emergency effect compared with single region emergency dispatching.

\subsubsection{Effects of different vulnerabilities $v_{I}$}

We investigate the impacts of the different path vulnerabilities by changing the values of $v_{l}$ that connect the rescue points in the single region (Table 3 ).

In the single region emergency scenario, with an increase in the emergency path's vulnerability, the total emergency scheduling quantity has a slight increase to 5.1, the minimum emergency costs remain virtually unchanged, and the generalized emergency costs of the entire network greatly increase from 39.5 to 58.6. To relieve the shortage condition, the rescue points should be appropriately increased to allocate more emergency funds if the path vulnerability increases. In short, different vulnerabilities for the emergency path will result in an emergency scheduling quantity change, which further affects the minimum relief costs and the generalized emergency costs at each crisis. Increasing the robustness of the emergency path can effectively improve the emergency response efficiency.

In the cross-regional emergency scenario, with an increase in the emergency path's vulnerability, the emergency scheduling quantity has a slight increase 0.004 , but its total emergency scheduling quantity is less than the single region mode. Additionally, the minimum relief costs increase from 1190.38 to 1190.39 , and this value is 0.5 larger than in the single region. Finally, its generalized emergency costs are all less than the single ones
$(39.5>35.9,58.6>54.6)$, and the degree of generalized emergency costs' decrease is more than 3.5. Therefore, with the increase in the emergency path's vulnerability, the cross-regional emergency coordination mode is better than the single region emergency dispatching. When the crossregional emergency dispatching network reaches equilibrium, the total scheduling of petroleum decreases. However, the generalized emergency costs are less than the single region scenario.

\subsubsection{Effects of different maximum emergency response capabilities $s_{i}$}

We investigate the effects of different maximum emergency response capabilities and compare the emergency costs by establishing relatively larger and smaller emergency capabilities (Table 4).

In the single region emergency scenario, the results show that when the maximum emergency capability on line 2 is increased, two effects happen. First, the scheduling quantity in region B is increased. Furthermore, when the maximum emergency capability is decreased, the scheduling quantity also decreases. Specifically, the quantity of petroleum on line 2 significantly decreases from 5.0 to 3.0. Second, the minimum relief costs after reaching the network equilibrium will incrementally increase from 595.0 to 597.0. Therefore, the increase in the maximum emergency capacity will result in a larger emergency scheduling quantity for the entire network and smaller minimum costs for the rescue point for the corresponding crisis point. Otherwise, the results will be the opposite. For petroleum supply, we should increase the maximum emergency capacity to effectively promote a more flexible emergency response mode.

Table 3 Effect of the path's vulnerability in different scenarios

\begin{tabular}{|c|c|c|c|c|c|c|}
\hline \multirow[t]{2}{*}{$v_{l}$} & \multicolumn{2}{|c|}{ Emergency scheduling quantity } & \multicolumn{2}{|c|}{ The minimum relief cost } & \multicolumn{2}{|c|}{ Generalized emergency cost } \\
\hline & Single region & Cross-region & Single region & Cross-region & Single region & Cross-region \\
\hline$v_{1}=v_{2}=1$ & 5.1 & 5.0 & 1189.9 & 1190.4 & 39.5 & 35.9 \\
\hline$v_{1}=v_{2}=2$ & 5.1 & 5.0 & 1189.9 & 1190.4 & 58.6 & 54.6 \\
\hline
\end{tabular}


Table 4 Effect of path's maximum emergency capability of the supply point in different scenarios

\begin{tabular}{|c|c|c|c|c|c|c|}
\hline \multirow[t]{2}{*}{$s_{l}$} & \multicolumn{2}{|c|}{ Emergency scheduling quantity of petroleum } & \multicolumn{2}{|c|}{ The minimum relief cost } & \multicolumn{2}{|c|}{ Generalized emergency cost } \\
\hline & Single region & Cross-region & Single region & Cross-region & Single region & Cross-region \\
\hline$s_{2}=5$ & 5.0 & 4.9 & 595.0 & 595.4 & 39.5 & 35.9 \\
\hline$s_{2}=6$ & 6 & 5.9 & 594 & 595.1 & 45.0 & 40.0 \\
\hline$s_{2}=3$ & 3 & 3.1 & 597.0 & 596.6 & 59.3 & 28.0 \\
\hline
\end{tabular}

In the cross-regional emergency scenario, we witness three effects. First, when the maximum emergency capability on line 2 is changed, the change in the scheduling quantity in the cross-regional mode coincides with it in the single one. However, the variational change of cross-regional emergency dispatching is smaller. Second, when the maximum emergency capability is increased, the minimum emergency costs decrease, and these costs are greater than those in the single one. However, when the maximum emergency capability is decreased, the minimum emergency costs increase and the costs are less than the single mode. Third, the generalized emergency costs are less than the single mode. When the maximum emergency capability is decreased, the generalized emergency costs sharply decline. Furthermore, the increase in the maximum emergency capability leads to an argument with the petroleum emergency quantity. Then, the total network coordination quantity increases and the generalized emergency costs sharply decline. All of these show that the cross-regional emergency coordination mode is better than single region emergency dispatching.

\subsubsection{Effects of different linkages $r_{\text {I }}$}

We investigate the influence of regional links between the emergency agents on the network equilibrium and compare changes in emergency costs by setting different values of regional links (Table 5).

In the single region emergency scenario, the results show that as the links between rescue point B and transfer point 2 decrease, the emergency scheduling quantity of petroleum in this region decreases. However, the minimum relief costs and the general emergency costs both increase.
In addition, by setting $r_{2}=10$, the petroleum on that line increases to 5.0, while the minimum relief costs and the general emergency costs are reduced to 595.0 and 37.0, respectively. That finding means that the increase in links tends to result in more allocated petroleum and lower costs. Therefore, with an increase in cross-regional links, there should be more petroleum for an emergency, lower generalized emergency costs, and better emergency effects. Otherwise, the emergency effects will be weakened. There are two ways to reduce the emergency costs. One is increasing the coordination of the cross-regional emergency, and the other is technical innovations in the petroleum emergency.

In the cross-regional emergency scenario, with the decrease in links between rescue point B and transfer point 2 , the emergency scheduling quantity of petroleum in this region decreases, and this quantity is less than that in the single mode. The minimum relief costs and generalized emergency costs both increase under identical conditions. However, the generalized emergency costs sharply decline when the link between rescue point B and transfer point 2 increases, which is less than the single one. Compared with the single region emergency dispatching mode, the crossregional mode has lower costs and more capacity. Although the minimum relief costs increased slightly the cross-regional emergency coordination mode is better than the single region emergency dispatching.

\subsubsection{Effects of different crisis point's vulnerabilities $v_{w}$ at the crisis points}

The vulnerability at the crisis point reflects the comprehensive capacity of the petroleum emergency, which

Table 5 Effect of the regional linkage in different scenarios

\begin{tabular}{|c|c|c|c|c|c|c|}
\hline \multirow[t]{2}{*}{$r_{l}$} & \multicolumn{2}{|c|}{ Emergency scheduling of petroleum } & \multicolumn{2}{|c|}{ The minimum relief cost } & \multicolumn{2}{|c|}{ Generalized emergency cost } \\
\hline & Single region & Cross-region & Single region & Cross-region & Single region & Cross-region \\
\hline$r_{2}=4$ & 5.0 & 4.9 & 595.0 & 595.4 & 39.5 & 35.9 \\
\hline$r_{2}=1$ & 5.0 & 4.9 & 595.0 & 595.4 & 40.9 & 36.8 \\
\hline$r_{2}=10$ & 5.0 & 5.0 & 595.0 & 595.4 & 37.0 & 27.5 \\
\hline
\end{tabular}


determines the minimum relief costs and the demand at the crisis point. The effect of different vulnerabilities on the supernetwork is analysed as follows (Table 6).

In the single region emergency scenario, the results show that the increase in minimum relief costs in the crisis point 1 will decrease the emergency scheduling quantity of petroleum in region $\mathrm{A}$ from 10.1 to 9.9 , increase the minimum relief costs and decrease the generalized emergency costs of the entire network from 39.5 to 36.9 . The above analysis indicates that if the vulnerabilities of crisis points change, the allocated petroleum on the path will be significantly increased, while the minimum relief costs and the general emergency costs will also surge. However, we are setting $v_{w 1}=v_{w 2}=1$, which indicates that the crisis point has a strong robustness. Afterwards, the minimum relief costs and the petroleum quantity at crisis points 1 and 2 sharply decrease to 597.0 and 5.0, respectively, and the generalized emergency costs decline to 23.0. This finding shows that if the region's response capacity for relieving petroleum shortages is strong, the generalized emergency costs will barely be affected by the petroleum shortages. Ultimately, for regional petroleum emergency scheduling, with the increasing vulnerability of petroleum demand, there will be larger demand and a greater minimum cost for the petroleum emergency. Otherwise, they will barely be affected by the shortage of petroleum. In the cross-regional emergency scenario, with the increase in the minimum relief costs at the crisis point 1 , the emergency scheduling quantity of petroleum in region A increases. Furthermore, when the vulnerability of crisis points 1 and 2 reach a maximum, the emergency scheduling quantity of petroleum increases instead. The change of minimum emergency costs is consistent with the changing direction of the minimum relief costs. When the vulnerability of crisis points 1 and 2 increase or decrease, the generalized emergency costs both decrease. However, while the vulnerability of crisis points 1 and 2 reach a minimum, the generalized emergency costs in the cross-regional mode is much higher than the single one $(33.5 \gg 23.0)$. In short, the more vulnerable the crisis point is, the more we need the cross-regional emergency dispatching coordination model. If the crisis point has a higher robustness, the single region emergency mode is obviously more efficient.

\subsection{Analysis of the treatment with different attributes on emergency dispatch}

For cross-regional emergency scheduling, we compare the influences of path vulnerability, maximum emergency capability and links on the equilibrium of the cross-regional emergency scheduling network. Thus, we propose the optimal resource scheduling scheme for the cross-regional petroleum emergency.

\subsubsection{Effects of the cross-regional path vulnerability $v_{I}(I=5,6,7,8)$}

The study of the influence of different vulnerabilities on the cross-regional emergency scheduling optimization is conducted by $v_{6}=1$. In addition, the simulation results are shown in Table 7.

When the vulnerability on line 6 is descending, the quantity of scheduled petroleum in region $\mathrm{A}$ and $\mathrm{B}$ are all maintained. Additionally, the generalized emergency costs decrease from 35.9 to 33.9. These results demonstrate that reducing the path vulnerability will improve the overall advantage of cross-regional emergency scheduling. Furthermore, by setting $v_{5}=v_{6}=v_{7}=v_{8}=5$ and increasing the vulnerability on the four lines, we find that the scheduled petroleum in regions A and B increase to 5.0 and 5.0, respectively, and the total increases from 9.9 to 9.9 . Likewise, the generalized emergency costs increase to 55.1. These analysis results show that with an increase in path vulnerability, more petroleum demand and higher scheduling costs will be generated to balance the crossregional network. Therefore, it is no longer beneficial to use the cross-regional emergency scheduling in this scenario.

In short, for multi-regional petroleum emergency conditions, the cross-regional emergency scheduling will be of practical value under small path vulnerability and strong emergency capacity. Compared with the single region, we

Table 6 Effect of the crisis point's vulnerability in different scenarios

\begin{tabular}{|c|c|c|c|c|c|c|}
\hline \multirow[t]{2}{*}{$v_{w}$} & \multicolumn{2}{|c|}{ Emergency scheduling of petroleum } & \multicolumn{2}{|c|}{ The minimum relief cost } & \multicolumn{2}{|c|}{ Generalized emergency cost } \\
\hline & Single region & Cross-region & Single region & Cross-region & Single region & Cross-region \\
\hline$v_{w 1}=200$ & 10.1 & 9.9 & 1189.9 & 1190.4 & 39.5 & 35.9 \\
\hline$v_{w 1}=1000$ & 9.9 & 9.9 & 1990.1 & 1989.9 & 36.9 & 28.6 \\
\hline$v_{w 1}=1$ & 5.0 & 10.1 & 597.0 & 591.9 & 23.0 & 33.5 \\
\hline$v_{w 2}=1$ & & & & & & \\
\hline
\end{tabular}


Table 7 Effect of the path's vulnerability

\begin{tabular}{lllll}
\hline$v_{l}$ & $\begin{array}{l}\text { Emergency scheduling of petroleum } \\
\text { in region A }\end{array}$ & $\begin{array}{l}\text { Emergency scheduling of petroleum } \\
\text { in region B }\end{array}$ & $\begin{array}{l}\text { Total amount of scheduled } \\
\text { petroleum }\end{array}$ & $\begin{array}{l}\text { Generalized } \\
\text { emergency cost }\end{array}$ \\
\hline$v_{6}=3$ & 5.0 & 4.9 & 9.9 & 35.9 \\
$v_{6}=1$ & 5.0 & 4.9 & 9.9 & 33.9 \\
$v_{5}=v_{6}=5$ & 5.0 & 5.0 & 9.9 & 55.1 \\
$v_{7}=v_{8}=5$ & & & & \\
\hline
\end{tabular}

find that there are two ways to solve the emergency problem of path vulnerability. The first is to increase the robustness of the emergency path, and the other is selecting the cross-regional emergency plan. Obviously, the second is more convenient and effective.

\subsubsection{Effects of the maximum emergency capability of line $s_{I}(I=5,6,7,8)$}

By reducing the maximum emergency response capability of lines in the cross-regional emergency scheduling network, we investigate the changes of the total petroleum quantity and the emergency costs (Table 8).

When reducing the maximum emergency capability of cross-regional lines, we find that the scheduled petroleum in region $\mathrm{A}$ increases from 5.0 to 5.1 and in region $\mathrm{B}$ it increases from 4.9 to 5.1 , which leads to an increase in total allocation from 9.9 to 10.2. Second, the generalized emergency costs increase from 35.9 to 38.0. That finding shows that decreasing the maximum emergency capability will reduce the benefits of cross-regional emergency scheduling in the supernetwork. By increasing the maximum emergency response capability of the four lines and setting $s_{5}=s_{6}=s_{7}=s_{8}=5$, the scheduled petroleum in regions $\mathrm{A}$ and $\mathrm{B}$ increases to 5.0 and 5.1, and the total scheduling increases from 9.9 to 10.1. Furthermore, the generalized emergency costs decrease from 35.9 to 33.3. The advantages of a cross-regional scheduling network will be enhanced with an increase in the emergency response capability. However, when the maximum emergency response capability continues to increase to 10 or 100 , the cross-regional emergency scheduling network should be maintained, except for the decreasing generalized emergency costs. All of these phenomena indicate that when the change of maximum emergency capability exceeds the demand, there will be no effects on the scheduling programme.

Consequently, reducing the maximum emergency capability of cross-regional lines will weaken the advantages of cross-regional scheduling. Otherwise, the emergency capability in the supernetwork will increase to the maximum demand. Therefore, it is beneficial to make the best of cross-regional scheduling by ensuring the smooth flow of emergency paths and maximum emergency capabilities.

\subsubsection{Effects of different linkages between cross-regional lines $r_{I}(I=5,6,7,8)$}

Finally, we study the influence of different linkages between the lines in different regions on the network resource optimization (Table 9).

Table 8 Effect of the maximum emergency capacity of lines

\begin{tabular}{|c|c|c|c|c|}
\hline$s_{l}$ & $\begin{array}{l}\text { Emergency scheduling of } \\
\text { petroleum in region A }\end{array}$ & $\begin{array}{l}\text { Emergency scheduling of } \\
\text { petroleum in region B }\end{array}$ & $\begin{array}{l}\text { Total amount of scheduled } \\
\text { petroleum }\end{array}$ & $\begin{array}{l}\text { Generalized } \\
\text { emergency cost }\end{array}$ \\
\hline $\begin{array}{l}s_{5}=5, s_{6}=1 \\
s_{7}=3, s_{8}=2\end{array}$ & 5.0 & 4.9 & 9.9 & 35.9 \\
\hline $\begin{array}{l}s_{5}=s_{6}=1 \\
s_{7}=s_{8}=1\end{array}$ & 5.1 & 5.1 & 10.1 & 38.0 \\
\hline $\begin{array}{l}s_{5}=s_{6}=5 \\
s_{7}=s_{8}=5\end{array}$ & 5.0 & 5.1 & 10.1 & 33.3 \\
\hline $\begin{array}{l}s_{5}=s_{6}=10 \\
s_{7}=s_{8}=10\end{array}$ & 5.1 & 5.0 & 10.1 & 33.2 \\
\hline $\begin{array}{l}s_{5}=s_{6}=100 \\
s_{7}=s_{8}=100\end{array}$ & 5.1 & 5.0 & 10.1 & 33.2 \\
\hline
\end{tabular}


Table 9 Effect of the regional linkage between regions

\begin{tabular}{lllll}
\hline$r_{l}$ & $\begin{array}{l}\text { Emergency scheduling of refined oil in } \\
\text { region A }\end{array}$ & $\begin{array}{l}\text { Emergency scheduling of refined } \\
\text { oil in B }\end{array}$ & $\begin{array}{l}\text { Total amount of } \\
\text { refined oil }\end{array}$ & $\begin{array}{l}\text { Generalized emergency } \\
\text { cost }\end{array}$ \\
\hline Basic data & 5.0 & 4.9 & 9.9 & 35.9 \\
$r_{5}=r_{6}=1$ & 5.0 & 4.9 & 9.9 & 38.9 \\
$r_{7}=r_{8}=1$ & & & & 35.2 \\
$r_{5}=7$ & 5.0 & 4.9 & 9.9 & 34.5 \\
$r_{5}=9$ & 5.0 & 4.9 & 9.9 & \\
\hline
\end{tabular}

After reducing the links between cross-regional lines, the scheduled and the generalized emergency costs increase, and the generalized emergency costs also greatly increase. Therefore, this increase will reduce the advantages of cross-regional emergency scheduling. Conversely, if we increase the links between lines $\left(r_{5}=7\right)$, the scheduled petroleum on this path increases from 1.4 to 1.6, and the total scheduling remain unchanged. However, the generalized emergency costs decrease to 35.2. When we continue to increase the links to 9 on line 5, the emergency costs will be reduced from 35.9 to 34.5. This finding means that increasing the path's cross-regional emergency scheduling links is beneficial for cross-regional emergency scheduling.

\section{Conclusions and recommendations}

In this paper, the supernetwork theory is used to describe the cross-regional emergency scheduling mechanism for petroleum shortages. Based on the analysis of a cross-regional emergency scheduling mechanism, we proposed a supernetwork model to find the optimal strategy in a petroleum shortage emergency. Considering the emergency costs and time, we establish the entire emergency response targets and design the optimal rescue behaviour. To solve the problem, an effective algorithm was used to simulate and conduct the parameter analysis. The simulation comparison results show that the cross-regional petroleum emergency scheduling pattern is superior with low emergency costs and high petroleum output. The advantages of the cross-regional scheduling will be strengthened with an increase in emergency response capabilities and regional associations, but they will disappear after achieving the maximum emergency capability. However, the single-regional emergency dispatch is preferable for conditions with little vulnerability. These conclusions have certain guiding significance for the formulation of relevant emergency policies and provide useful thoughts for decision-making, regardless of cross-regional or single-regional scenarios.
In a future study, we will further consider the impact of different regional emergency agents and emergency responses on the entire cross-regional emergency scheduling supernetwork of petroleum. Furthermore, we will also attempt to explore the dynamic optimization of a cross-regional emergency supernetwork and consider more special features of petroleum emergency management, such as the unexpected event of a petroleum shortage and uncertainties in the petroleum emergency demand.

Based on the research results, we offer certain corresponding recommendations from the aspects of emergency agents and emergency paths. First, the government should clarify some influential factors for a petroleum shortage, such as the smooth flow of emergency paths and the maximum emergency capabilities, and propose cross-regional emergency scheduling for petroleum emergency management based on these influential factors. As discussed in this paper, the strategy of petroleum emergency scheduling depends on the change of influential factors. For example, the vulnerabilities in the cross-regional link and in the regional links determine whether the petroleum emergency scheduling is single region or cross-regions. Targeting the different ranges of influence factors, the corresponding petroleum emergency management strategies are provided. In addition, China should conscientiously summarize the emergency measures for worldwide petroleum shortages and select the appropriate petroleum emergency programme that adapts to the realistic national conditions based on scenarios and countermeasures.

Second, the petroleum transportation management should broaden the transportation modes of petroleum, which could improve the anti-risk ability of the petroleum transportation network. The diversity of transport modes will increase the robustness of the emergency path, thus enhancing the regional emergency capability of petroleum. In China, the transportation strategy puts a high reliance on the railways while pipelines lag. Therefore, it is advisable to develop pipeline transportation and fundamentally change the current pattern of petroleum transportation.

Third, the government should improve the regional association and establish a regional synergistic mechanism 
that is conducive to constructing a collaborative emergency response framework for petroleum. In this study, we find that cross-regional petroleum emergency scheduling dependent on both the modes of transport but is also affected by the regional association. Therefore, China should improve the cross-regional emergency coordination mechanism, including the information, the materials and the incentive protection mechanism, to enhance the regional response flexibility of emergency warnings.

Acknowledgements The work in this paper was supported by the Fundamental Research Funds for the Central Universities (Grant No. 2014XT06).

Open Access This article is distributed under the terms of the Creative Commons Attribution 4.0 International License (http://creative commons.org/licenses/by/4.0/), which permits unrestricted use, distribution, and reproduction in any medium, provided you give appropriate credit to the original author(s) and the source, provide a link to the Creative Commons license, and indicate if changes were made.

\section{References}

Abdalla RM, Alharbi SM. The role of GIS technology in assessing critical infrastructure resiliency during emergencies in the City of Jeddah, Saudi Arabia. Int $J$ Emerg Manag. 2017;13(2):183-91.

Ambituuni A, Amezaga JM, Werner D. Risk assessment of petroleum product transportation by road: a framework for regulatory improvement. Saf Sci. 2015;79:324-35. https://doi.org/10.1016/ j.ssci.2015.06.022.

Asia Pacific Energy Research Center. Oil supply security and emergency policy in the APEC region. SESSION 1 - OIL AND GAS SECURITY STUDIES, The 1st APEC Oil and Gas Security Network Forum. Kitakyushu City, Japan. 2015.

Bai Y, Zhou P, Tian L, Meng F. Desirable strategic petroleum reserves policies in response to supply uncertainty: a stochastic analysis. Appl Energy. 2016;162:1523-9. https://doi.org/10. 1016/j.apenergy.2015.04.025.

British Petroleum (BP). British Petroleum (BP) annual statistical review in 2015. 2015. http://www.bp.com/content/dam/bp-coun try/zh-cn/Publications/2015SR/Statistical\%20Review\%20of\% 20World\%20Energy\%202015\%20CN\%20Final\%2020150617. pdf. Accessed 20 May 2017.

Chen JJ, Wu YQ, Feng SK. Research on the emergency resources allocation of oil products. J Univ Electron Sci Technol China (Social Sciences Edition). 2013;4:49-53 (in Chinese).

Fernandes LJ, Relvas S, Barbosa-Póvoa AP. Strategic network design of downstream petroleum supply chains: single versus multientity participation. Chem Eng Res Des. 2013;91:1557-87. https://doi.org/10.1016/j.cherd.2013.05.028.

Iakovou ET. An interactive multi objective model for the strategic maritime transportation of petroleum products: risk analysis and routing. Saf Sci. 2001;39:19-29. https://doi.org/10.1016/S09257535(01)00022-4.

Jiang HM. An analysis of CNP's emergency oil product supply during the Wenchuan earthquake. Int Pet Econ. 2008;7:63-68+92 (in Chinese)

Jiang HM. Research on the application of "the Shortest Path" problem in contingency supply of refined oil. J Southwest Pet Univ (Social Sciences Edition). 2013;1:9-14 (in Chinese).
Jiang HM, Zhang GH, Luo Q, Guo B. Construction of a refined oil logistics and distribution system. J Southwest Pet Univ (Social Sciences Edition). 2014;2:7-14 (in Chinese).

Kazemi Y, Szmerekovsky J. Modeling downstream petroleum supply chain: the importance of multi-mode transportation to strategic planning. Transp Res Part E: Logist Transp Rev. 2015;83:111-25. https://doi.org/10.1016/j.tre.2015.09.004.

Liao F, Arentze T, Molin E, et al. Effects of land-use transport scenarios on travel patterns: a multi-state supernetwork application. Transportation. 2015;1:1-25. https://doi.org/10.1007/ s11116-015-9616-Z.

Liu Z, Nagurney A. Supply chain networks with global outsourcing and quick-response production under demand and cost uncertainty. Ann Oper Res. 2013;208:251-89. https://doi.org/10.1007/ s10479-011-1006-0.

Lu ZG, Gao P. Petro China's primary oil product logistics today, challenges and solutions. Sino-Global Energy. 2016;5(1):14-20 (in Chinese).

Lyu Y, Yang Y, Wei W, An W. Risk assessment and emergency response of oil spill for offshore oil activity. Int Oil Spill Conf Proc. 2014;1:545-60. https://doi.org/10.7901/2169-3358-20141-299545.1.

MirHassani SA. An operational planning model for petroleum products logistics under uncertainty. Appl Math Comput. 2008;196:744-51. https://doi.org/10.1016/j.amc.2007.07.006.

Nagurney A. On the relationship between supply chain and transportation network equilibria: a supernetwork equivalence with computations. Transp Res Part E: Logist Transp Rev. 2006;42:293-316. https://doi.org/10.1016/j.tre.2005.02.001.

Nagurney A, Dong J, Zhang D. A supply chain network equilibrium model. Transp Res Part E: Logist Transp Rev. 2002;38:281-303. https://doi.org/10.1016/S1366-5545(01)00020-5.

NetEase Financial News. Many cities suffered the "diesel shortage" in China. 2001. http://news.163.com/special/Youhuang/ (in Chinese). Accessed 20 May 2017.

Oliveira F, Nunes PM, Blajberg R. A framework for crude oil scheduling in an integrated terminal-refinery system under supply uncertainty. Eur J Oper Res. 2016;252:635-45. https:// doi.org/10.1016/j.ejor.2016.01.034.

Othman SB, Zgaya H, Dotoli M. An agent-based decision support system for resources' scheduling in emergency supply chains. Control Eng Pract. 2017;59:27-43. https://doi.org/10.1016/j. conengprac.2016.11.014

Sha HE, Yao HU, Linyuan W, Lin Z. The thought of cloud technology in the emergency management of petroleum industry. Ind Saf Environ Prot. 2017;1:026 (in Chinese).

Sharma RK, Gurjar BR, Singhal AV, et al. Automation of emergency response for petroleum oil storage terminals. Saf Sci. 2015;72:262-73. https://doi.org/10.1016/j.ssci.2014.09.019.

Wang ZP, Wang ZT. The supernetwork theory and its applications. Beijing: The Science Publishing Company; 2008 (in Chinese).

Wang N, Xu W, Xu Z, Shao W. A survey on supernetwork research: theory and applications. Control Conf (CCC). 2016;35:1202-6. https://doi.org/10.1109/ChiCC.2016.7553250.

Yamada T, Imai K, Nakamura T. A supply chain-transport supernetwork equilibrium model with the behaviour of freight carriers. Transp Res Part E: Logist Transp Rev. 2011;47:887-907. https:// doi.org/10.1016/j.tre.2011.05.009.

Zhang H, Liang Y, Liao Q, Yan X, Shen Y, Zhao Y. A three-stage stochastic programming method for LNG supply system infrastructure development and inventory routing in demanding countries. Energy. 2017;133:424-42.

Zhu L, Cao J. Supernetwork optimization of emergency resources allocation under disaster risk. Chin J Manag Sci. 2012;6:141-8 (in Chinese). 
Zhu L, Cao J. Urban agglomeration coordination for emergency resources allocation: a supernetwork structure perspective. Manag Rev. 2015;7:207-17 (in Chinese).
Zhu L, Du YQ. Supernetwork model of urban agglomeration coordination for emergency resources allocation. Math Pract Theory. 2015;16:27-37 (in Chinese). 